\title{
Molecular Insight into the Pathway to Crystallization of Aluminium
}

\author{
Supporting Information \\ Caroline Desgranges and Jerome Delhommelle \\ Department of Chemical Engineering, 301, Main Street South, \\ University of South Carolina, Columbia SC 29201
}

\section{Simulation methods of crystal nucleation and growth}

We choose the interaction potential parametrized by Jasper et al. J. Phys. Chem. B, 109, 3915 (2005) to model aluminium. This embedded-atom (EAM) potential was validated against density-functional theory (DFT) results for aluminium clusters and nanoparticles. This potential was then used in Gibbs Ensemble Monte Carlo simulations in the Gibbs Ensemble to predict the critical point of Al (Bhatt et al. J. Am. Chem. Soc., 128, 4224 (2006)) and the normal boiling point in good agreement with the experimental value (Bhatt et al. J. Phys. Chem. B, 110, 26135 (2006)). Using thermodynamic integration, Bhatt et al. estimated a melting point of $1122 \pm 13 \mathrm{~K}$ at ambient pressure. In this work, we carry out simulations of crystal nucleation and growth in a supercooled liquid of aluminium at fixed pressure $(P=1 \mathrm{~atm})$ and at fixed supercooling (i.e. at $T=897.6 \mathrm{~K}$ - a temperature $20 \%$ below the melting point estimated by Bhatt et al.).

\subsection{Simulation of the nucleation step}

For a supercooling of $20 \%$, crystal nucleation is an activated process. It can be studied by combining the umbrella sampling technique with Monte Carlo or molecular dynamics simulations (see e.g. ten Wolde et al. J. Chem. Phys., 104, 9932 (1996)). In the umbrella sampling technique, we perform a non-Boltzmann sampling by applying a so-called umbrella bias potential. The bias potential is a harmonic function of the global order parameter $Q_{6}$ introduced by Steinhardt et al. Phys. Rev. B, 28, 784 (1983). $Q_{6}$ is a measure of the overall crystallinity of the system: it is equal to 0 for a liquid and takes similar values for all the structures we came across in this work ( 0.575 for the face centered cubic - fcc- structure, 0.485 for the hexagonal close packed - hcp - structure, 0.511 for the body centered cubic - bcc - structure and 0.663 for the icosahedral - Ih structure). Therefore, using a function of $Q_{6}$ as the bias potential will not favor the formation of a specific polymorph.

In this work, we combine the umbrella sampling technique with the Hybrid Monte Carlo (HMC) method introduced by Mehlig et al. Phys. Rev. B, 45, 679 (1992). Each HMC step consists of either a molecular dynamics trajectory of 10 timesteps of 2 fs ( $67 \%$ of the HMC steps) or of a Monte Carlo volume change (33\% of the HMC steps - the amplitude of the volume change is adjusted so that $50 \%$ of these moves are accepted). Velocities are drawn from a Gaussian distribution at the beginning of each molecular dynamics trajectory and the equations of motion are integrated with a velocity-Verlet integrator.

\subsection{Simulation of the growth step}

Once we have formed the critical nucleus, we study its evolution in the absence of the bias potential. We first embed the system of 3,000 particles containing a critical nucleus in a supercooled liquid of 27,000 particles and obtain a system containing a total of 30,000 particles.

We then equilibrate this system according to the following two successive steps: - the central region of 3,000 particles is kept fixed and we let the rest of the system relax during a HMC simulation in the NPT ensemble;

- once the rest of the system has relaxed, we carry out a HMC simulation of the whole system (still in the NPT ensemble) while applying the umbrella sampling bias potential to the central subsystem of 3,000 particles. 
Every 1,000 HMC steps, we store an equilibrated configuration of the system and use this configuration as a starting point of an unbiased "trajectory" (i.e. simulations for which we have switched off the umbrella sampling potential) in the NPT ensemble. This unbiased (or free) trajectory is generated using the stochastic molecular dynamics simulation method proposed by Attard, J. Chem. Phys., 116, 9616 (2002), for the isothermal-isobaric ensemble. This method consists of deterministic time steps (using Newton's equations of motion), alternated with stochastic steps based upon the Boltzmann distribution (it is very similar to the popular method proposed by Andersen, J. Chem. Phys., 72, 2384 (1980)). During each deterministic time step, we integrate the equation of motion with a velocity-Verlet integrator and a timestep of $2 \mathrm{fs}$. We found that this combined Monte Carlo and molecular dynamics technique was more efficient than the HMC method to study the free evolution of the critical nuclei. We store 15 equilibrated configurations containing a critical nucleus of aluminium and thus generate 15 free evolutions of a critical nucleus of aluminium. We observe the dissolution of the nucleus in the surrounding liquid for 8 of them and the growth of the nucleus in the remaining 7 free evolutions. The 8:7 ratio, close to the ideal 5:5 ratio, expected for a critical nucleus, demonstrate that the crystal nuclei we have formed are critical nuclei.

\section{Identification of the structural identity of the particles}

We describe here how we analyze the structure of the crystal nucleus and how we identify the structural identity of each particle: icosahedral (Ih), face centred cubic (fcc), hexagonal close packed (hcp) or body centred cubic (bcc) of each particle.

\subsection{Distinguishing between solid-like and liquid-like particles}

We first briefly outline how we distinguish between solid-like and liquid-like particles (this is done according to the method proposed by ten Wolde et al. J. Chem. Phys., 104, 9932 (1996)). For a given molecule $i$, local orientational order parameters $\bar{q}_{l m}(i)(m=-l \ldots l)$ are defined according to

$$
\bar{q}_{l m}(i)=\frac{\sum_{j} Y_{l m}\left(\hat{\mathbf{r}}_{i j}\right) \alpha\left(r_{i j}\right)}{\sum_{j} \alpha\left(r_{i j}\right)}
$$

where $Y_{l m}\left(\hat{\mathbf{r}}_{i j}\right)$ is a spherical harmonics, $\alpha\left(r_{i j}\right)$ a weight function $\left(\alpha\left(r_{i j}\right)=\left(r_{i j}-r_{q}\right)^{2}\right.$ for $r_{i j}<r_{q}$ and $\alpha\left(r_{i j}\right)=0$ elsewhere). $j$ denotes a molecule which belongs to the first coordination shell of molecule $i$ and $\mathbf{r}_{i j}$ the vector joining the centers of mass of molecules $i$ and $j$ ( $r_{i j}$ stands for the norm of this vector and $\hat{\mathbf{r}}_{i j}$ the corresponding unit vector). $r_{q}=3.5 \AA$, corresponds to the first minimum of $\mathrm{g}(\mathrm{r})$ in the fcc crystal of $\mathrm{Al}$.

We now define the normalized 13-dimensional $(m=-6, \ldots, 6)$ complex vector $\mathbf{q}_{6}(i)$ of components $\tilde{q}_{6 m}(i)$ defined as

$$
\tilde{q}_{6 m}(i)=\frac{\bar{q}_{6 m}(i)}{\left(\sum_{m=-6}^{6}\left|\bar{q}_{6 m}(i)\right|^{2}\right)^{1 / 2}}
$$

The dot product of the vectors $\mathbf{q}_{6}$ of two neighboring molecules $i$ and $j$ is

$$
\mathbf{q}_{6}(i) \cdot \mathbf{q}_{6}(j)=\sum_{m=-6}^{6} \tilde{q}_{6 m}(i) \tilde{q}_{6 m}^{*}(j)
$$

Two molecules are considered to be connected if $\mathbf{q}_{6}(i) \cdot \mathbf{q}_{6}(j)$ is larger than 0.5 i.e. if their local order parameters are correlated. We find that the number of connections per molecule rarely exceeds 8 in the liquid phase (less than $1 \%$ are identified as solid-like) and is seldom below 8 in both solid phases. A molecule connected to at least 8 molecules of its first neighbors is considered as solid-like and liquid-like otherwise. 


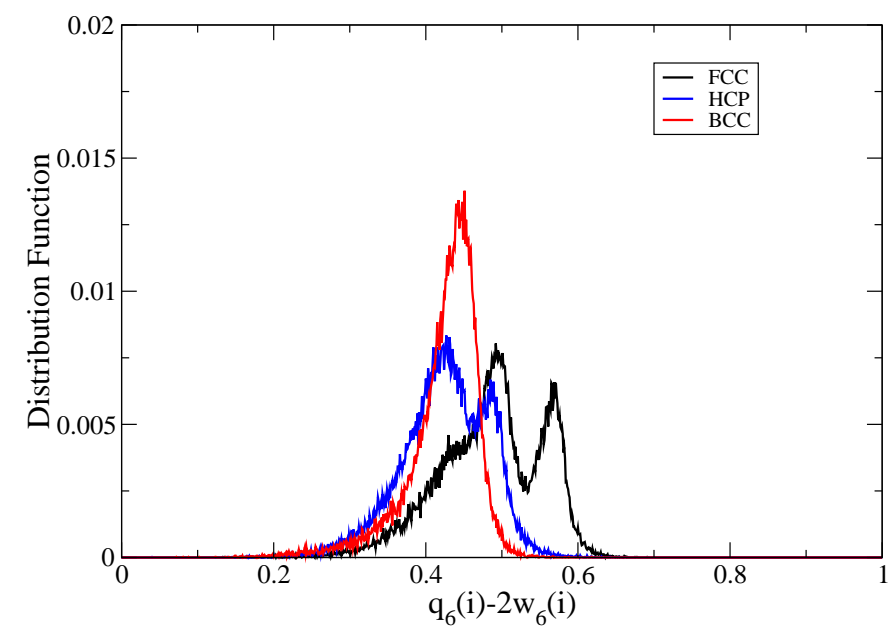

FIG. 1: Distribution of $q_{6}-2 \hat{w}_{6}$ for the fcc, bcc and hcp crystals at $T=897.6 \mathrm{~K}$ and $P=1 \mathrm{~atm}$

\subsection{Distinguishing between Ih-like, fcc-like, bcc-like and hcp-like particles}

Once we have determined which particles are solid-like, we need to assign a specific structure to each of these solid particles i.e. Ih-like, fcc-like, bcc-like and hcp-like particles.

We first determine which particles are Ih-like. For this purpose, we evaluate the order parameter $\hat{w}_{6}$ from the local order parameter $\bar{q}_{6 m}(i)$ as

$$
\hat{w}_{6}(i)=\frac{w_{6}(i)}{\left[\sum_{m=-6}^{6}\left|\bar{q}_{6 m}(i)\right|^{2}\right]^{3 / 2}}
$$

where

$$
w_{6}(i)=\sum_{\substack{m_{1}, m_{2}, m_{3} \\
m_{1}+m_{2}+m_{3}=0}}\left(\begin{array}{ccc}
6 & 6 & 6 \\
m_{1} & m_{2} & m_{3}
\end{array}\right) \times \bar{q}_{6 m_{1}}(i) \bar{q}_{6 m_{2}}(i) \bar{q}_{6 m_{3}}(i)
$$

We then define a new order parameter $I P$ as the linear combination of the two order parameters $q_{6}$ and $\hat{w}_{6}$

$$
I P=q_{6}-2 \hat{w}_{6}
$$

This linear combination takes a value of 1 for a perfect Ih structure, of 0.6 for a perfect fcc structure, of 0.51 for a perfect hcp structure and 0.49 for a perfect bcc structure. At a temperature of $897.6 \mathrm{~K}$, we obtain the distribution functions of $I P$ plotted in Fig. 1 for the fcc, hcp and bcc crystals. As can be seen from this plot, IP is always less than 0.7 for the three fcc, hcp and bcc structures. Hence, we decide to identify as Ih-like all particles (whether they are solid-like or liquid-like) for which we have a value of $I P$ greater than 0.7 . 


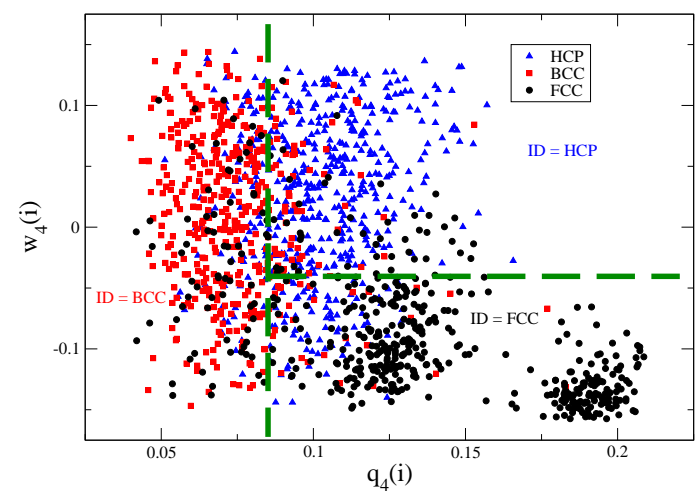

FIG. 2: Rules used to determine whether a particle is fcc-like, bcc-like or hcp-like.

Once the Ih-like particles have been identified, we use two other order parameters $q_{4}$ and $\hat{w}_{4}$ to distinguish between fcc-like, bcc-like and hcp-like particles. $q_{4}$ and $\hat{w}_{4}$ are defined from the local order parameters $\bar{q}_{l m}(i)$ as

$$
\begin{aligned}
& q_{4}(i)=\left[\frac{4 \pi}{9} \sum_{m=-4}^{4}\left|\bar{q}_{4 m}(i)\right|^{2}\right]^{1 / 2} \\
& \hat{w}_{4}(i)=\frac{w_{4}(i)}{\left[\sum_{m=-4}^{4}\left|\bar{q}_{4 m}(i)\right|^{2}\right]^{3 / 2}}
\end{aligned}
$$

where

$$
w_{4}(i)=\sum_{\substack{m_{1}, m_{2}, m_{3} \\
m_{1}+m_{2}+m_{3}=0}}\left(\begin{array}{ccc}
4 & 4 & 4 \\
m_{1} & m_{2} & m_{3}
\end{array}\right) \times \bar{q}_{4 m_{1}}(i) \bar{q}_{4 m_{2}}(i) \bar{q}_{4 m_{3}}(i)
$$

We compute the distributions for the two local order parameters $q_{4}$ and $\hat{w}_{4}$ for the three crystalline structures fcc, bcc and hcp at the temperature of $897.6 \mathrm{~K}$.

On the basis of these distributions, we identify a particle $i$ as

- fcc-like if $q_{4}(i)>0.105$ and $\hat{w}_{4}(i)<-0.04$,

- bcc-like if $q_{4}(i)<0.085$,

- hcp-like if $q_{4}(i)>0.085$ and $\hat{w}_{4}(i)>-0.04$.

We summarize these rules in Fig. 2.

An estimate of the accuracy of the rules used to determine whether a particle is fcc-like, bcc-like or hcp-like can be obtained by applying these rules to configurations of the three solid phases equilibrated at $T=897.6 \mathrm{~K}$ and $P=1 \mathrm{~atm}$. On average (over 1,000 configurations obtained during a HMC simulations of the fcc crystal), we identify $73 \pm 3 \%$ of the particles as fcc-like. We obtain a similar accuracy for the bcc and hcp crystals. 